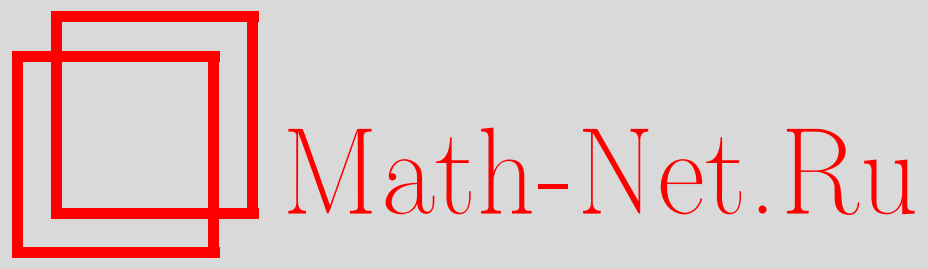

В. А. Никишкин, Об оценках решений краевых задач для эллиптических систем в слое, Функи. анализ и его прил., 2011, том 45, выпуск 2, 60-70

DOI: https://doi.org/10.4213/faa3036

Использование Общероссийского математического портала MathNet.Ru подразумевает, что вы прочитали и согласны с пользовательским соглашением

http://www.mathnet.ru/rus/agreement

Параметры загрузки:

IP : 35.173 .219 .12

26 апреля 2023 г., 14:49:59

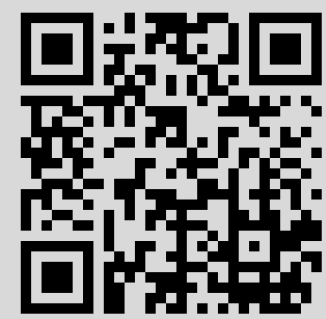


Функиионалъный анализ и его приложения

2011, т. 45, вып. 2, с. 60-70

УДК 517.956.223

\title{
Об оценках решений краевых задач для эллиптических систем в слое*
}

\author{
(c) 2011. В. А. Никишкин
}

Посвящается памяти моего учителя Владимира Александровича Кондратвева

\begin{abstract}
Рассматриваются общие краевые задачи для эллиптических систем в слое, т.е. в области

$$
\Pi=\left\{\left(x^{\prime}, x_{n}\right) \in \mathbb{R}^{n} \mid x^{\prime} \in \mathbb{R}^{n-1}, x_{n} \in(a, b)\right\}, \quad-\infty<a<b<+\infty, n \geqslant 3 .
$$

Предполагается выполненным условие Лопатинского. Установлены теоремы о разрешимости, получены оценки решений.
\end{abstract}

Общие краевые задачи для эллиптических систем в областях с гладкой границей рассматривались, в частности, в работах [9]-[12].

Фундаментальная статья В. А. Кондратьева [13] положила начало исследованиям эллиптических краевых задач в областях, границы которых содержат особенности - углы, конические точки, ребра и т. п. Общая теория краевых задач для эллиптических систем в таких областях содержится в [8].

В работе [2] рассматриваются решения обыкновенных дифференциальных уравнений

$$
\frac{1}{i} \frac{d u}{d t}-A u=f
$$

где $u(t)$ - функции со значениями в некотором банаховом пространстве, и изучается поведение решений при $t \rightarrow+\infty$. Основные предположения этой работы касаются области регулярности в комплексной $\lambda$-плоскости резольвенты

$$
R(\lambda ; A)=(\lambda I-A)^{-1}
$$

оператора $A$. Получена экспоненциальная асимптотика решений. В частности, изучена область регулярности резольвенты и получена экспоненциальная асимптотика решений эллиптических краевых задач в цилиндрических областях.

Постановка задачи. Введем обозначения $D_{\mu}=-i \partial / \partial x_{\mu}(\mu=1, \ldots, n)$, $x^{\prime}=\left(x_{1}, \ldots, x_{n-1}\right), x=\left(x^{\prime}, x_{n}\right), \xi^{\prime}=\left(\xi_{1}, \ldots, \xi_{n-1}\right), \xi=\left(\xi^{\prime}, \xi_{n}\right), \zeta=\left(\zeta_{1}, \ldots, \zeta_{n-1}\right)$, $p=\left(p_{1}, \ldots, p_{n-1}\right), \Gamma^{+}=\left\{x \in \mathbb{R}^{n} \mid x^{\prime} \in \mathbb{R}^{n-1}, x_{n}=a\right\}, \Gamma^{-}=\left\{x \in \mathbb{R}^{n} \mid x^{\prime} \in\right.$ $\left.\mathbb{R}^{n-1}, x_{n}=b\right\}$.

В области П рассмотрим систему уравнений

$$
A\left(D_{1}, \ldots, D_{n}\right) w(x)=\tilde{f}(x) \quad(x \in \Pi) .
$$

*Исследования поддержаны РФФИ, грант 08-01-00819-а, и Министерством образования и науки, грант АВП РНП 2.1.1/7161. 
Здесь $w$ и $\tilde{f}$ - векторы-столбцы высоты $N$, а $A-$ квадратная матрица порядка $N$, состоящая из линейных дифференциальных операторов с постоянными комплексными коэффициентами. Через $k$ обозначим наивысший порядок операторов, входящих в $A\left(D_{1}, \ldots, D_{n}\right)$. В дальнейшем будет предполагаться, что $N k$ четно.

На границе $\Gamma^{ \pm}$ставятся условия

$$
\left.B_{\nu}^{ \pm}\left(D_{1}, \ldots, D_{n}\right) w(x)\right|_{x \in \Gamma^{ \pm}}=\tilde{g}_{\nu}^{ \pm}\left(x^{\prime}\right) \quad(\nu=1, \ldots, N k / 2) .
$$

Здесь $B_{\nu}^{ \pm}\left(D_{1}, \ldots, D_{n}\right)$ - строки длины $N$, состоящие из линейных дифференциальных операторов с постоянными комплексными коэффициентами. Через $m_{\nu}^{ \pm}\left(m_{\nu}^{ \pm}<k\right)$ обозначим наивысший порядок операторов в строке.

При замене $D_{j}$ на $\xi_{j}(j=1, \ldots, n)$ операторы $A$ и $B_{\nu}^{ \pm}$переходят в полиномиальные матрицу $A(\xi)$ и строки $B_{\nu}^{ \pm}(\xi)$. Обозначим через $A_{0}(\xi)$ и $B_{\nu 0}^{ \pm}(\xi)$ главные части матрицы $A(\xi)$ и строк $B_{\nu}^{ \pm}(\xi)$, составленные из однородных полиномов степеней $k$ и $m_{\nu}^{ \pm}$.

Рассмотрим прямое произведение $N$ пространств Соболева $H^{s}(\Pi)$ с нормой

$$
\|v\|_{s}=\|v\|_{s, \Pi}=\left(\int_{\mathbb{R}_{\zeta}^{n-1}} \int_{a}^{b}\left(\sum_{q+t=0}^{s}|\zeta|^{2 t}\left|D_{n}^{q} F v\right|^{2}\right) d \zeta d x_{n}\right)^{1 / 2},
$$

где $F v$ - преобразование Фурье по $x^{\prime}$, задаваемое формулой

$$
F v=\frac{1}{(2 \pi)^{n / 2}} \int_{\mathbb{R}^{n-1}} e^{-i \zeta x^{\prime}} v d x^{\prime} .
$$

Рассмотрим также прямые произведения $N k / 2$ пространств $H^{s}\left(\Gamma^{ \pm}\right)$для дробных $s$ с нормой

$$
\|v\|_{s}=\|v\|_{s, \Gamma^{ \pm}}=\left(\int_{\mathbb{R}_{\zeta}^{n-1}}\left(1+|\zeta|^{2 s}\right)|F v|^{2} d \zeta\right)^{1 / 2} .
$$

Обозначим эти пространства тоже через $H^{s}(\Pi)$ и $H^{s}\left(\Gamma^{ \pm}\right)$. Квадрат нормы вектора из $H^{s}$ равен сумме квадратов норм его компонент.

Матрица $A$ является эллиптической $\left(\operatorname{det} A_{0}(\xi) \neq 0\right.$ при $\left.\xi \neq 0, \xi \in \mathbb{R}^{n}\right)$ и правильной, т. е. уравнение $\operatorname{det} A_{0}\left(\xi^{\prime}, \lambda\right)=0$ относительно $\lambda$ имеет (при ненулевых $\left.\xi^{\prime}\right) N k / 2$ решений в верхней полуплоскости и столько же в нижней.

Условие 1 (условие Лопатинского). Введем две локальные системы координат с началом в точках $a$ и $b$ соответственно и осью $y$, направленной внутрь отрезка $[a, b]$. Рассмотрим пространство решений системы

$$
A_{0}\left(\xi^{\prime},-i \frac{d}{d y}\right) v\left(\xi^{\prime}, y\right)=0, \quad y>0,
$$

стремящихся к нулю при $y \rightarrow+\infty$ вместе со всеми производными. Условие заключается в том, что в этом пространстве система имеет единственное решение (равное нулю), удовлетворяющее начальному условию

$$
\left.B_{0 \nu}^{+}\left(\xi^{\prime},-i \frac{d}{d y}\right) v\left(\xi^{\prime}, y\right)\right|_{y=0}=0 \quad(\nu=1, \ldots, N k / 2)
$$


(соответственно

$$
\left.\left.B_{0 \nu}^{-}\left(\xi^{\prime},-i \frac{d}{d y}\right) v\left(\xi^{\prime}, y\right)\right|_{y=0}=0 \quad(\nu=1, \ldots, N k / 2)\right) .
$$

Обозначим через $M(\gamma, p)$ плоскость в комплексном пространстве $\mathbb{C}^{n-1}$ параметров $\xi^{\prime}$, задаваемую соотношениями

$$
\operatorname{Im} \xi_{j}=p_{j}, \quad p_{j}=\mathrm{const} \quad(j=1, \ldots, n-1), \quad \sum_{j=1}^{n-1}\left(p_{j}\right)^{2}=\gamma^{2}, \quad \gamma=\mathrm{const} .
$$

Предполагается также, что выполнено следующее

Условие 2. Задача

$$
\begin{aligned}
A\left(\xi^{\prime}, D_{n}\right) u\left(\xi^{\prime}, x_{n}\right) & =0, & & \\
\left.B_{\nu}^{+}\left(\xi^{\prime}, D_{n}\right) u\left(\xi^{\prime}, x_{n}\right)\right|_{x_{n}=a} & =0 & & (\nu=1, \ldots, N k / 2), \\
\left.B_{\nu}^{-}\left(\xi^{\prime}, D_{n}\right) u\left(\xi^{\prime}, x_{n}\right)\right|_{x_{n}=b} & =0 & & (\nu=1, \ldots, N k / 2)
\end{aligned}
$$

имеет только тривиальное решение для $\xi^{\prime} \in M(\gamma, p)$.

Основным результатом настоящей работы является

Теорема 1. Пусть $\gamma \geqslant 0, l \geqslant k$, и пусть в задаче (1)-(2) $A\left(D_{1}, \ldots, D_{n}\right)$ - правильная эллиптическая система операторов, причем выполнены условия 1 и 2. Тогда для любых функиий $\tilde{f}(x), \tilde{g}_{\nu}^{+}\left(x^{\prime}\right), \tilde{g}_{\nu}^{-}\left(x^{\prime}\right), \operatorname{maкux,~что~} V_{\gamma}\left(x^{\prime}\right) \tilde{f} \in$ $H^{l-k}(\Pi), V_{\gamma}\left(x^{\prime}\right) \tilde{g}_{\nu}^{+} \in H^{l-m_{\nu}^{+}-1 / 2}\left(\Gamma^{+}\right), V_{\gamma}\left(x^{\prime}\right) \tilde{g}_{\nu}^{-} \in H^{l-m_{\nu}^{-}-1 / 2}\left(\Gamma^{-}\right)$, существует одно и только одно решение $w(x)$ задачи $(1)-(2)$, такое, что $V_{\gamma}\left(x^{\prime}\right) w \in H^{l}(\Pi)$. При этом имеет место оценка

$$
\left\|V_{\gamma} w\right\|_{l} \leqslant C\left(\left\|V_{\gamma} \tilde{f}\right\|_{l-k}+\sum_{\nu=1}^{N k / 2}\left\|V_{\gamma} \tilde{g}_{\nu}^{+}\right\|_{l-m_{\nu}^{+}-1 / 2}+\sum_{\nu=1}^{N k / 2}\left\|V_{\gamma} \tilde{g}_{\nu}^{-}\right\|_{l-m_{\nu}^{-}-1 / 2}\right),
$$

где $V_{\gamma}\left(x^{\prime}\right)=\left(\gamma\left|x^{\prime}\right|\right)^{(3-n) / 2} I_{(n-3) / 2}\left(\gamma\left|x^{\prime}\right|\right), I_{s}(z)$ - модифицированная функиия Бесселя [14], а константа $C$ не зависит от $w, \tilde{f}, \tilde{g}_{\nu}^{+}, \tilde{g}_{\nu}^{-}$.

Для доказательства теоремы 1 потребуется теорема 2, доказательство которой с небольшими изменениями аналогично доказательству соответствующих утверждений для систем с одним параметром в работе [1].

Теорема 2. Пусть $l \geqslant k$ и $A\left(\xi^{\prime}, D_{n}\right)$ - правильная матрица, состоящая из дифференциальных операторов с постоянными комплексными коэффициентами, такая, что $\operatorname{det} A_{0}(\xi) \neq 0$ при $\xi \neq 0, \xi \in \mathbb{R}^{n}$. Для задачи (3)-(5) выполнено условие Лопатинского. Тогда при $\left|\xi^{\prime}\right| \geqslant \xi_{0}$ (где $\xi_{0}$ - достаточно болъшое положительное число) для любой функиии $f \in H^{l-k}(a, b)$ и любъх $g_{\nu}^{+}\left(\xi^{\prime}\right), g_{\nu}^{-}\left(\xi^{\prime}\right)$ $(\nu=1, \ldots, N k / 2)$ существует одно и только одно решение задачи

$$
\begin{aligned}
A\left(\xi^{\prime}, D_{n}\right) u\left(\xi^{\prime}, x_{n}\right) & =f\left(\xi^{\prime}, x_{n}\right), & & \\
\left.B_{\nu}^{+}\left(\xi^{\prime}, D_{n}\right) u\left(\xi^{\prime}, x_{n}\right)\right|_{x_{n}=a} & =g_{\nu}^{+}\left(\xi^{\prime}\right) & & (\nu=1, \ldots, N k / 2), \\
\left.B_{\nu}^{-}\left(\xi^{\prime}, D_{n}\right) u\left(\xi^{\prime}, x_{n}\right)\right|_{x_{n}=b} & =g_{\nu}^{-}\left(\xi^{\prime}\right) & & (\nu=1, \ldots, N k / 2),
\end{aligned}
$$


принадлежсащее $H^{l}(a, b)$. При этом имеет место оценка

$$
\begin{aligned}
\|u\|_{l}+\left|\xi^{\prime}\right|^{l}\|u\|_{0} \leqslant C\left(\|f\|_{l-k}+\left|\xi^{\prime}\right|^{l-k}\|f\|_{0}\right. & +\sum_{\nu=1}^{N k / 2}\left(1+\left|\xi^{\prime}\right|^{l-m_{\nu}^{+}-1 / 2}\right)\left|g_{\nu}^{+}\right| \\
& \left.+\sum_{\nu=1}^{N k / 2}\left(1+\left|\xi^{\prime}\right|^{l-m_{\nu}^{-}-1 / 2}\right)\left|g_{\nu}^{-}\right|\right),
\end{aligned}
$$

где константа $C$ не зависит от $u, f, g_{\nu}, \xi^{\prime}$, но зависит от $\xi_{0}$.

Замечание 1 [2]. Подставим в операторы (7)-(9) $e^{i \theta_{j}} \xi_{j}$ вместо $\xi_{j}(j=$ $1, \ldots, n-1)$. Тогда если $\theta_{j}$ или $\pi-\theta_{j}(j=1, \ldots, n-1)$ вещественны и достаточно малы, то для полученных операторов по-прежнему будут выполнены все условия теоремы 2. Следовательно, утверждение теоремы 2 верно, если $\left|\xi^{\prime}\right| \geqslant \xi_{0}$, $\left|\arg \left( \pm \xi_{j}\right)\right| \leqslant \delta$ (где $j=1, \ldots, n-1, \xi_{0}$ - достаточно большое положительное число, а $\delta$ достаточно мало).

Замечание 2. Рассмотрим операторную вектор-функцию

$$
U\left(\xi^{\prime}\right)=\left(A\left(\xi^{\prime}, D_{n}\right), B_{\nu}^{+}\left(\xi^{\prime}, D_{n}\right), B_{\nu}^{-}\left(\xi^{\prime}, D_{n}\right)\right),
$$

которая действует из $H^{l}(a, b)$ в $H^{l-k}(a, b) \times \mathbb{R}^{N k}$. По теореме 2 и замечанию 1 , если $\left|\xi^{\prime}\right| \geqslant \xi_{0},\left|\arg \left( \pm \xi_{j}\right)\right| \leqslant \delta$ (где $j=1, \ldots, n-1, \xi_{0}-$ достаточно большое положительное число, а $\delta$ достаточно мало), то существует ограниченный оператор $R\left(\xi^{\prime}\right)$ (резольвента задачи (7)-(9)), который действует из $H^{l-k}(a, b) \times \mathbb{R}^{N k}$ в $H^{l}(a, b)$, такой, что

$$
U\left(\xi^{\prime}\right) R\left(\xi^{\prime}\right)=E
$$

Обозначим через

$$
y_{\eta}\left(\xi^{\prime}, x_{n}\right)=\left(y_{1 \eta}\left(\xi^{\prime}, x_{n}\right), \ldots, y_{N \eta}\left(\xi^{\prime}, x_{n}\right)\right) \quad(\eta=1, \ldots, N k)
$$

фундаментальную систему решений системы (3), определенную начальными условиями $y_{\eta}^{(\mu-1)}\left(\xi^{\prime}, 0\right)=\delta_{\mu, \eta}(\mu=1, \ldots, k)$, в которых при каждом $\eta$ только одно из чисел $\delta_{\mu, \eta}$ равно 1 , а остальные равны 0.

Коэффициенты оператора в (3) являются полиномами от параметров $\xi^{\prime}$, поэтому из [3] (гл. 1, §8, задача 7) следует, что функции из фундаментальной системы решений (11) для любого фиксированного $x_{n} \in[a, b]$ являются целыми аналитическими функциями параметров $\xi^{\prime}$. Обозначим через $Y\left(\xi^{\prime}, x_{n}\right)=$ $\left(y_{\eta}^{(\mu-1)}\left(\xi^{\prime}, x_{n}\right)\right)$ матрицу этой фундаментальной системы.

Для функции $f\left(\xi^{\prime}, x_{n}\right)$, не равной нулю тождественно, определим частное решение $u_{0}\left(\xi^{\prime}, x_{n}\right)$ системы $(7)$ по формуле

$$
u_{0}\left(\xi^{\prime}, x_{n}\right)=Y\left(\xi^{\prime}, x_{n}\right) \int_{a}^{x_{n}} Y^{-1}\left(\xi^{\prime}, t\right) f\left(\xi^{\prime}, t\right) d t .
$$

Таким образом, решение $u\left(\xi^{\prime}, x_{n}\right)$ задачи $(7)-(9)$ можно записать в виде $u=$ $u_{0}+u_{1}$, где $u_{1}\left(\xi^{\prime}, x_{n}\right)$ удовлетворяет системе

$$
\begin{array}{ccc}
A\left(\xi^{\prime}, D_{n}\right) u_{1}\left(\xi^{\prime}, x_{n}\right) & =0, & \\
\left.B_{\nu}^{+}\left(\xi^{\prime}, D_{n}\right)\left(u_{1}\left(\xi^{\prime}, x_{n}\right)+u_{0}\left(\xi^{\prime}, x_{n}\right)\right)\right|_{x_{n}=a}=g_{\nu}^{+}\left(\xi^{\prime}\right) & & (\nu=1, \ldots, N k / 2), \\
\left.B_{\nu}^{-}\left(\xi^{\prime}, D_{n}\right)\left(u_{1}\left(\xi^{\prime}, x_{n}\right)+u_{0}\left(\xi^{\prime}, x_{n}\right)\right)\right|_{x_{n}=b}=g_{\nu}^{-}\left(\xi^{\prime}\right) & (\nu=1, \ldots, N k / 2) .
\end{array}
$$


Для $u_{1}\left(\xi^{\prime}, x_{n}\right)$ справедливо представление

$$
u_{1}\left(\xi^{\prime}, x_{n}\right)=\sum_{\eta=1}^{N k} C_{\eta}\left(\xi^{\prime}\right) y_{\eta}\left(\xi^{\prime}, x_{n}\right)
$$

здесь $C_{\eta}\left(\xi^{\prime}\right)$ определяются после подстановки (16) в (13)-(15). Решение будет единственным тогда и только тогда, когда определитель системы для $C_{\eta}\left(\xi^{\prime}\right)$

$$
\left|\begin{array}{ccc}
B_{1}^{+} y_{1}(a) & \ldots & B_{1}^{+} y_{N k}(a) \\
\vdots & \ldots & \vdots \\
B_{N k / 2}^{+} y_{1}(a) & \ldots & B_{N k / 2}^{+} y_{N k}(a) \\
B_{1}^{-} y_{1}(b) & \ldots & B_{1}^{-} y_{N k}(b) \\
\vdots & \ldots & \vdots \\
B_{N k / 2}^{-} y_{1}(b) & \ldots & B_{N k / 2}^{-} y_{N k}(b)
\end{array}\right|
$$

отличен от нуля. Этот определитель также является целой аналитической функцией параметров $\xi^{\prime}$ (отличной от тождественного нуля).

Отсюда следует, что резольвента задачи $(7)-(9) R\left(\xi^{\prime}\right)$ является мероморфной функцией, а на множестве $M(\gamma, p)$ она будет аналитической функцией параметров $\xi^{\prime}$ вследствие условия 2 .

Замечание 3. Рассмотрим область, ограниченную поверхностями $M(\gamma, p)$ и $\left\{\xi^{\prime} \in \mathbb{C}^{n}:\left|\xi^{\prime}\right|=\xi_{0}\right\}$, где $\xi_{0}$ достаточно большое. На границе этой области $R\left(\xi^{\prime}\right)$ является аналитической. Из [4, теорема 1, гл. 4, §15] следует аналитичность $R\left(\xi^{\prime}\right)$ в цилиндре, ограниченном $M(\gamma, p)$.

Доказательство теоремы 1. Для каждого положительного $R$ рассмотрим срезающую функцию $\sigma_{R}\left(x^{\prime}\right)$, обладающую следующими свойствами [5]:

(1) $0 \leqslant \sigma_{R}\left(x^{\prime}\right) \leqslant 1$ для $x^{\prime} \in \mathbb{R}^{n-1}, \sigma_{R}\left(x^{\prime}\right)=1$ для $\left|x^{\prime}\right| \leqslant R, \sigma_{R}\left(x^{\prime}\right)=0$ для $\left|x^{\prime}\right| \geqslant 2 R$;

(2) существует константа $C$, не зависящая от $R$, такая, что неравенство $\left|\left(\partial^{\alpha} / \partial x^{\prime \alpha}\right) \sigma_{R}\left(x^{\prime}\right)\right| \leqslant C R^{-|\alpha|}$ выполняется для всех $x^{\prime} \in \mathbb{R}^{n-1}$ и $|\alpha| \leqslant l$.

Сначала теорема 1 доказывается для $\sigma_{R}\left(x^{\prime}\right) \tilde{f}(x), \sigma_{R}\left(x^{\prime}\right) \tilde{g}_{\nu}^{+}\left(x^{\prime}\right), \sigma_{R}\left(x^{\prime}\right) \tilde{g}_{\nu}^{-}\left(x^{\prime}\right)$, затем с использованием оценки (6) осуществляется предельный переход при $R \rightarrow \infty$.

Далее $\sigma_{R}\left(x^{\prime}\right) \tilde{f}(x), \sigma_{R}\left(x^{\prime}\right) \tilde{g}_{\nu}^{+}\left(x^{\prime}\right), \sigma_{R}\left(x^{\prime}\right) \tilde{g}_{\nu}^{-}\left(x^{\prime}\right)$ вновь будут обозначаться через $\tilde{f}(x), \tilde{g}_{\nu}^{+}\left(x^{\prime}\right), \tilde{g}_{\nu}^{-}\left(x^{\prime}\right)$, и в новых обозначениях пусть $f\left(\xi^{\prime}, x_{n}\right), g_{\nu}^{+}\left(\xi^{\prime}\right)$ и $g_{\nu}^{-}\left(\xi^{\prime}\right)-$ преобразование Фурье по $x^{\prime}$ функций $\tilde{f}(x), \tilde{g}_{\nu}^{+}\left(x^{\prime}\right)$ и $\tilde{g}_{\nu}^{-}\left(x^{\prime}\right)$.

Рассмотрим задачу (7)-(9). По теореме 2 и замечанию 1 при $\xi^{\prime} \in M(\gamma, p)$, таких, что $\left|\xi^{\prime}\right| \geqslant \xi_{0}$ (где $\xi_{0}$ - достаточно большое положительное число), существует единственное решение задачи (7)-(9), принадлежащее $H^{l}(a, b)$. При этом имеет место оценка (10), где константа $C$ не зависит от $u, f, g_{\nu}^{ \pm}, \xi^{\prime}$, но зависит oT $\xi_{0}$.

Из представлений $(12),(16)$ следует, что для $\xi^{\prime} \in M(\gamma, p)$, таких, что $\left|\xi^{\prime}\right| \leqslant \xi_{0}$, решение задачи $(7)-(9)$, лежащее в $H^{l}(a, b)$, удовлетворяет неравенству

$$
\|u\|_{l} \leqslant C\left(\|f\|_{l-k}+\sum_{\nu=1}^{N k / 2}\left|g_{\nu}^{+}\right|+\sum_{\nu=1}^{N k / 2}\left|g_{\nu}^{-}\right|\right) .
$$


Отсюда получается оценка $(10)$ для $\xi^{\prime} \in M(\gamma, p)$, таких, что $\left|\xi^{\prime}\right| \leqslant \xi_{0}$.

Заметим, что вместо оценки (10) мы можем применять эквивалентное неравенство

$$
\begin{aligned}
\|u\|_{l}^{2}+\left|\xi^{\prime}\right|^{2 l}\|u\|_{0}^{2} \leqslant C\left(\|f\|_{l-k}^{2}+\left|\xi^{\prime}\right|^{2 l-2 k}\|f\|_{0}^{2}\right. & +\sum_{\nu=1}^{N k / 2}\left(1+\left|\xi^{\prime}\right|^{2 l-2 m_{\nu}^{+}-1}\right)\left|g_{\nu}^{+}\right|^{2} \\
& \left.+\sum_{\nu=1}^{N k / 2}\left(1+\left|\xi^{\prime}\right|^{2 l-2 m_{\nu}^{-}-1}\right)\left|g_{\nu}^{-}\right|^{2}\right)
\end{aligned}
$$

где $C$ не зависит от $p$, принадлежащего шару с центром в начале координат радиуса $\gamma$.

Рассмотрим функцию

$$
w=\frac{1}{(2 \pi)^{n / 2}} \int_{M(\gamma, p)} e^{i x^{\prime} \xi^{\prime}} u d \operatorname{Re} \xi^{\prime} .
$$

Для финитных по $x^{\prime}$ правых частей системы (1)-(2) этот интеграл сходится в среднем (квадратическом) в силу неравенства (17) и условий теоремы 1 и по теореме Планшереля определяет функцию $w$, такую, что (после применения равенства Парсеваля) для нее справедлива оценка

$$
\left\|e^{-p x^{\prime}} w\right\|_{l}^{2} \leqslant C\left(\left\|e^{-p x^{\prime}} \tilde{f}\right\|_{l-k}^{2}+\sum_{\nu=1}^{N k / 2}\left\|e^{-p x^{\prime}} \tilde{g}_{\nu}^{+}\right\|_{l-m_{\nu}^{+}-1 / 2}^{2}+\sum_{\nu=1}^{N k / 2}\left\|e^{-p x^{\prime}} \tilde{g}_{\nu}^{-}\right\|_{l-m_{\nu}-1 / 2}^{2}\right),
$$

где константа $C$ не зависит от $w, \tilde{f}, \tilde{g}_{\nu}^{+}, \tilde{g}_{\nu}^{-}$.

Вследствие замечания 3 можно утверждать, что $w$ не зависит от $p$, если $p$ принадлежит сфере $E_{\gamma}$ радиуса $\gamma$ с центром в начале координат. Действительно, пусть $p_{1}^{(1)}$ и $p_{1}^{(2)}$ - две точки на $E_{\gamma}$. Соединим их ломаной, целиком лежащей в шаре, ограниченном сферой $E_{\gamma}$, каждое звено которой параллельно одной из координатных осей, и рассмотрим любое из этих звеньев. Не ограничивая общности, можем считать, что меняется только первая координата на отрезке $\left[p_{1}^{(1)}, p_{1}^{(2)}\right]$. Положим

$$
w_{1}=\int_{-\infty+i p_{2}^{(1)}}^{\infty+i p_{2}^{(1)}} \cdots \int_{-\infty+i p_{n-1}^{(1)}}^{\infty+i p_{n-1}^{(1)}} e^{i\left(x_{2} \xi_{2}+\cdots+x_{n-1} \xi_{n-1}\right)} u\left(\xi^{\prime}, x_{n}\right) d \operatorname{Re} \xi_{2} \cdots d \operatorname{Re} \xi_{n-1} .
$$

По формуле Коши для $L>0$ имеет место равенство

$$
\begin{gathered}
\int_{-L+i p_{1}^{(1)}}^{L+i p_{1}^{(1)}} e^{i x_{1} \xi_{1}} w_{1} d \operatorname{Re} \xi_{1}=i \int_{-L+i p_{1}^{(1)}}^{-L+i p_{1}^{(2)}} e^{i x_{1} \xi_{1}} w_{1} d \operatorname{Im} \xi_{1} \\
\int_{-L+i p_{1}^{(2)}}^{L+i p_{1}^{(2)}} e^{i x_{1} \xi_{1}} w_{1} d \operatorname{Re} \xi_{1}+i \int_{L+i p_{1}^{(2)}}^{L+i p_{1}^{(1)}} e^{i x_{1} \xi_{1}} w_{1} d \operatorname{Im} \xi_{1} .
\end{gathered}
$$

Следуя работе [6, теорема 2.2], покажем, что первый и третий интегралы в правой части равенства (19) стремятся к нулю по некоторой последовательности значений $L \rightarrow+\infty$. Из теорем вложения и оценки (17) следует оценка модуля 
$u\left(\xi^{\prime}, x_{n}\right)$, откуда и получаем требуемое утверждение, так как только наличие зависимости от $x_{n}$ отличает этот случай от рассмотренного в доказательстве теоремы 2.2 в [6].

Проинтегрируем неравенство (18) по переменным $\left(p_{1}, \ldots, p_{n-1}\right)$ по сфере $E_{\gamma}$. Зависимость от $\left(p_{1}, \ldots, p_{n-1}\right)$ есть только в экспоненте, поэтому нам достаточно вычислить интеграл

$$
\int_{E_{\gamma}} e^{-p_{1} x_{1}-\cdots-p_{n-1} x_{n-1}} d p_{1} \cdots d p_{n-1}
$$

Нетрудно показать, что этот интеграл зависит только от $\left|x^{\prime}\right|$. Сравнивая разложение в ряд рассматриваемого интеграла с разложением в ряд модифицированной функции Бесселя, получаем, что этот интеграл равен

$$
2 \pi^{(n-1) / 2} \gamma^{n-1}\left(\gamma\left|x^{\prime}\right|\right)^{(3-n) / 2} I_{(n-3) / 2}\left(\gamma\left|x^{\prime}\right|\right) ;
$$

здесь $I_{s}(z)$ - модифицированная функция Бесселя.

Опишем теперь подробнее предельный переход для решений задачи (1)-(2) с финитными правыми частями $\sigma_{R}\left(x^{\prime}\right) \tilde{f}(x), \sigma_{R}\left(x^{\prime}\right) \tilde{g}_{\nu}^{+}\left(x^{\prime}\right), \sigma_{R}\left(x^{\prime}\right) \tilde{g}_{\nu}^{-}\left(x^{\prime}\right)$ при $R \rightarrow \infty$. Выберем последовательность $R_{s} \rightarrow \infty$ при $s \rightarrow \infty$. Соответствующая последовательность решений задачи $(1)-(2) w_{R_{s}}$ является фундаментальной в рассматриваемых пространствах $H^{l}(\Pi)$ с весом $V_{\gamma}\left(x^{\prime}\right)$, что следует из оценки (6). Отсюда получаем существование предела - функции $w$, такой, что $V_{\gamma}\left(x^{\prime}\right) w \in H^{l}(\Pi)$. Используя слабую сходимость, получаем, что $w$ является решением задачи (1)-(2). Единственность решения задачи (1)-(2) в рассматриваемых пространствах следует из оценки (6).

Теорема 1 доказана.

Теорема 3. Пусть $\gamma_{2} \geqslant \gamma \geqslant \gamma_{1} \geqslant 0, l \geqslant k, u$ пусть в задаче (1)-(2) $A\left(D_{1}, \ldots, D_{n}\right)$ - правильная эллиптическая матрица операторов, причем въполнены условия 1 и 2 для всех таких $\gamma$. Если $V_{\gamma_{1}}\left(x^{\prime}\right) w \in H^{l}(\Pi), V_{\gamma_{2}}\left(x^{\prime}\right) \tilde{f} \in$ $H^{l-k}(\Pi), V_{\gamma_{2}}\left(x^{\prime}\right) \tilde{g}_{\nu}^{+} \in H^{l-m_{\nu}^{+}-1 / 2}\left(\Gamma^{+}\right), V_{\gamma_{2}}\left(x^{\prime}\right) \tilde{g}_{\nu}^{-} \in H^{l-m_{\nu}^{-}-1 / 2}\left(\Gamma^{-}\right)$, mo $V_{\gamma_{2}}\left(x^{\prime}\right) w$ $\in H^{l}(\Pi)$. При этом имеет место оценка

$$
\left\|V_{\gamma_{2}} w\right\|_{l} \leqslant C\left(\left\|V_{\gamma_{2}} \tilde{f}\right\|_{l-k}+\sum_{\nu=1}^{N k / 2}\left\|V_{\gamma_{2}} \tilde{g}_{\nu}^{+}\right\|_{l-m_{\nu}^{+}-1 / 2}+\sum_{\nu=1}^{N k / 2}\left\|V_{\gamma_{2}} \tilde{g}_{\nu}^{-}\right\|_{l-m_{\nu}^{-}-1 / 2}\right),
$$

где константа $C$ не зависит от $w, \tilde{f}, \tilde{g}_{\nu}^{+}, \tilde{g}_{\nu}^{-}$.

Доказательство проводится аналогично доказательству теоремы 2.2 из [6].

Теорема 4. Пусть $\gamma \geqslant 0, l \geqslant k$, и пусть в задаче $(1)-(2) A\left(D_{1}, \ldots, D_{n}\right)$ - правильная эллиптическая система операторов, причем выполнень условия 1 и 2. Тогда для любых функиий $\tilde{f}(x), \tilde{g}_{\nu}^{+}\left(x^{\prime}\right), \tilde{g}_{\nu}^{-}\left(x^{\prime}\right)$, maких, что е $\gamma\left|x^{\prime}\right| \tilde{f} \in$ $H^{l-2 m}(\Pi), e^{\gamma\left|x^{\prime}\right|} \tilde{g}_{\nu}^{+} \in H^{l-m_{\nu}^{+}-1 / 2}\left(\Gamma^{+}\right), e^{\gamma\left|x^{\prime}\right|} \tilde{g}_{\nu}^{-} \in H^{l-m_{\nu}^{-}-1 / 2}\left(\Gamma^{-}\right)$, cуществует одно и только одно решение $w(x)$ задачи (1)-(2), такое, что $e^{\gamma\left|x^{\prime}\right|} w \in H^{l}(\Pi)$. При этом имеет место оценка

$\left\|e^{\gamma\left|x^{\prime}\right|} w\right\|_{l} \leqslant C\left(\left\|e^{\gamma\left|x^{\prime}\right|} \tilde{f}\right\|_{l-k}+\sum_{\nu=1}^{N k / 2}\left\|e^{\gamma\left|x^{\prime}\right|} \tilde{g}_{\nu}^{+}\right\|_{l-m_{\nu}^{+}-1 / 2}+\sum_{\nu=1}^{N k / 2}\left\|e^{\gamma\left|x^{\prime}\right|} \tilde{g}_{\nu}^{-}\right\|_{l-m_{\nu}^{-}-1 / 2}\right)$,

где константа $C$ не зависит от $w, \tilde{f}, \tilde{g}_{\nu}^{+}, \tilde{g}_{\nu}^{-}$. 
Доказательство. Произведем в задаче (1)-(2) замену искомых функций $w$, положив $w(x)=v(x) \varphi\left(x^{\prime}\right)$, где $\varphi\left(x^{\prime}\right)=\left(1+\varepsilon\left|x^{\prime}\right|\right)^{(2-n) / 4}$. Используя формулу Лейбница, можем написать

$$
A\left(v(x) \varphi\left(x^{\prime}\right)\right)=\varphi\left(x^{\prime}\right) A v(x)+\ldots, \quad B_{\nu}^{ \pm}\left(v(x) \varphi\left(x^{\prime}\right)\right)=\varphi\left(x^{\prime}\right) B_{\nu}^{ \pm} v(x)+\ldots,
$$

где в членах, замененных многоточием, $\varphi\left(x^{\prime}\right)$ дифференцируется хотя бы один раз.

Умножим полученные уравнения и граничные условия для $v$ на $\left(\varphi\left(x^{\prime}\right)\right)^{-1}$. Тогда для $v$ получим задачу

$$
\begin{aligned}
A v(x)+\varepsilon \widetilde{A}(x) v(x) & =\left(\varphi\left(x^{\prime}\right)\right)^{-1} \tilde{f}(x) & & (x \in \Pi), \\
B_{\nu}^{ \pm} v(x)+\left.\varepsilon \widetilde{B}(x)_{\nu}^{ \pm} v(x)\right|_{x \in \Gamma^{ \pm}} & =\left(\varphi\left(x^{\prime}\right)\right)^{-1} \tilde{g}_{\nu}^{ \pm}\left(x^{\prime}\right) & & (\nu=1, \ldots, N k / 2),
\end{aligned}
$$

где $\widetilde{A}(x), \widetilde{B}(x)$ - операторы с бесконечно дифференцируемыми ограниченными коэффициентами, а правые части системы (20)-(21) удовлетворяют условиям теоремы 1. При достаточно малом $\varepsilon$ левые части соотношений определяют оператор с переменными коэффициентами, мало отличающийся по норме от обратимого оператора задачи (1)-(2) в теореме 1. Применение теоремы 1 завершает доказательство теоремы 4.

Переменные коэффициенты. Рассмотрим теперь задачу

$$
\begin{aligned}
A\left(x, D_{1}, \ldots, D_{n}\right) w(x) & =\tilde{f}(x) & & (x \in \Pi), \\
\left.B_{\nu}^{ \pm}\left(x, D_{1}, \ldots, D_{n}\right) w(x)\right|_{x \in \Gamma^{ \pm}} & =\tilde{g}_{\nu}^{ \pm}\left(x^{\prime}\right) & & (\nu=1, \ldots, N k / 2)
\end{aligned}
$$

с переменными бесконечно дифференцируемыми в $\bar{\Pi}$ коэффициентами. Будем предполагать, что в каждой точке $x \in \bar{\Pi}$ выполнено условие правильной эллиптичности, а в каждой точке $x \in \Gamma^{ \pm}-$условие Лопатинского. Предположим также выполненным условие равномерной по $x_{n} \in[a, b]$ стабилизации коэффициентов на бесконечности:

$$
\begin{aligned}
\lim _{x^{\prime} \rightarrow \infty} A\left(x, D_{1}, \ldots, D_{n}\right) & =A\left(D_{1}, \ldots, D_{n}\right), \\
\lim _{x^{\prime} \rightarrow \infty} B_{\nu}^{ \pm}\left(x, D_{1}, \ldots, D_{n}\right) & =B_{\nu}^{ \pm}\left(D_{1}, \ldots, D_{n}\right) .
\end{aligned}
$$

Здесь $A\left(D_{1}, \ldots, D_{n}\right), B_{\nu}^{ \pm}\left(D_{1}, \ldots, D_{n}\right)$ - операторы задачи $(1)-(2)$.

Используем определение нормально разрешимого оператора, данное в работе [15] на с. 51.

Теорема 5. Пусть для задачи (22)-(23) выполнены все перечисленные предположения, а для задачи (1)-(2) выполнены предположения теоремы 1 или теоремы 4. Тогда задача (22)-(23) нормально разрешима в тех же пространстваx.

Доказательство. Задача (22)-(23) является частным случаем задач, рассматриваемых в теореме 20.1.2 из [7]. В окрестности бесконечности $\left|x^{\prime}\right|>R$, где $R$ достаточно большое, применим условия стабилизации коэффициентов (24), (25) и теорему 1 или 4. Теорема 5 доказана.

Оценки модуля решения. Пусть $Q_{r} \times[a, b]-$ цилиндр радиуса $r>1$. Положим $\widetilde{G}_{0}=Q_{2} \times[a, b], G_{0}=Q_{3} \times[a, b], \widetilde{G}_{s}=\left(Q_{2+s} \backslash Q_{s+1}\right) \times[a, b]$ и $G_{s}=$ $\left(Q_{s+3} \backslash Q_{s}\right) \times[a, b](s=1,2, \ldots)$. 
Теорема 6. Пусть $\gamma_{1}>\gamma>0$, и пусть в задаче (1)-(2) $A\left(D_{1}, \ldots, D_{n}\right)-$ правильная эллиптическая система операторов, причем выполнены условия 1 u 2. Пусть $|\tilde{f}(x)| \leqslant K_{0} e^{-\gamma_{1}\left|x^{\prime}\right|}$ nри $x \in \bar{\Pi}, \tilde{g}_{\nu}^{+}\left(x^{\prime}\right) \equiv \tilde{g}_{\nu}^{-}\left(x^{\prime}\right) \equiv 0$. Тогда для решения $w(x)$ задачи (1)-(2) имеет место оченка

$$
|w(x)| \leqslant C K_{0} e^{-\gamma\left|x^{\prime}\right|} \quad(x \in \bar{\Pi}),
$$

где константа $C$ не зависит от $w$ и $\tilde{f}$.

Доказательство. По теореме 1.7 из [8] (неравенство (1.11)) для $p>1$ получаем

$$
\|w\|_{W_{p}^{k}\left(\widetilde{G}_{s}\right)} \leqslant C\left(\|\tilde{f}\|_{L_{p}\left(G_{s}\right)}+\|w\|_{L_{2}\left(G_{s}\right)}\right) \quad(s=0,1,2, \ldots),
$$

где $W_{p}^{k}$ - пространства, порожденные $L_{p}$-нормой $\left(W_{p}^{k}\right.$ при $p=2$ совпадают с пространствами Соболева)

$$
\|w\|_{W_{p}^{k}\left(\widetilde{G}_{s}\right)}=\left(\sum_{|\alpha| \leqslant k}\left(\left\|D^{\alpha} w\right\|_{L_{p}\left(\widetilde{G}_{s}\right)}\right)^{p}\right)^{1 / p},
$$

а константа $C$ не зависит от $\tilde{f}(x), w$ и $s(s=0,1,2, \ldots)$. Отсюда и из теорем вложения следует непрерывность $w(x)$ в $\bar{\Pi}$ и оценка

$$
|w(x)| \leqslant C\left(\|\tilde{f}\|_{L_{p}\left(G_{s}\right)}+\|w\|_{L_{2}\left(G_{s}\right)}\right) \quad(s=0,1,2, \ldots),
$$

где константа $C$ не зависит от $w$ и $s$. Умножим неравенство (26) на $e^{\gamma\left|x^{\prime}\right|}$. Увеличив константу $C$, отсюда получаем

$$
\left|e^{\gamma\left|x^{\prime}\right|} w(x)\right| \leqslant C\left(\left\|e^{\gamma\left|x^{\prime}\right|} \tilde{f}\right\|_{L_{p}\left(G_{s}\right)}+\left\|e^{\gamma\left|x^{\prime}\right|} w\right\|_{L_{2}\left(G_{s}\right)}\right) \quad\left(x \in \widetilde{G}_{s}, s=0,1,2, \ldots\right),
$$

где $C$ не зависит от $w$ и $s$.

Из оценок (27) и теоремы 4 следует утверждение теоремы 6.

В качестве примера рассмотрим задачу Дирихле для линейной системы теории упругости в слое $\Pi=\left\{\left(x^{\prime}, x_{3}\right) \in \mathbb{R}^{3} \mid x^{\prime} \in \mathbb{R}^{2}, x_{3} \in(0, \pi)\right\}$ :

$$
\begin{gathered}
\mu \Delta w+(\lambda+\mu) \operatorname{grad}(\operatorname{div} w)=\tilde{f} \quad(x \in \Pi), \\
w_{\Gamma^{ \pm}}=0,
\end{gathered}
$$

где $w=\left(w_{1}, w_{2}, w_{3}\right)$ - вектор смещения, а $\tilde{f}=\left(\tilde{f}_{1}, \tilde{f}_{2}, \tilde{f}_{3}\right)$ - плотность внешних сил, приложенных к слою. Постоянные $\lambda$ и $\mu$ удовлетворяют условию $\mu>0$, $\lambda+\mu>0$.

Система (3) в этом случае имеет вид

$$
\left\{\begin{array}{l}
\mu\left(\frac{\partial^{2} u_{1}}{\partial x_{3}^{2}}-\left(\xi_{1}^{2}+\xi_{2}^{2}\right) u_{1}\right)-(\lambda+\mu)\left(\xi_{1}^{2} u_{1}+\xi_{1} \xi_{2} u_{2}+i \xi_{1} \frac{\partial u_{3}}{\partial x_{3}}\right)=f_{1}, \\
\mu\left(\frac{\partial^{2} u_{2}}{\partial x_{3}^{2}}-\left(\xi_{1}^{2}+\xi_{2}^{2}\right) u_{2}\right)-(\lambda+\mu)\left(\xi_{1} \xi_{2} u_{1}+\xi_{2}^{2} u_{2}+i \xi_{2} \frac{\partial u_{3}}{\partial x_{3}}\right)=f_{2}, \\
\mu\left(\frac{\partial^{2} u_{3}}{\partial x_{3}^{2}}-\left(\xi_{1}^{2}+\xi_{2}^{2}\right) u_{3}\right)-(\lambda+\mu)\left(i \xi_{1} \frac{\partial u_{1}}{\partial x_{3}}+i \xi_{2} \frac{\partial u_{2}}{\partial x_{3}}-\frac{\partial^{2} u_{3}}{\partial x_{3}^{2}}\right)=f_{3} .
\end{array}\right.
$$

Пусть $\xi_{1}=\operatorname{Re} \xi_{1}+i p_{1}, \xi_{2}=\operatorname{Re} \xi_{2}+i p_{2}$.

Получим априорную оценку для $u \in W_{2}^{2}(0, \pi)$ и $f \in L_{2}(0, \pi)$. Оценим сначала первые производные функции $u$. Для этого умножим обе части системы (30) 
скалярно на $\bar{u}$, проинтегрируем по $[0, \pi]$ и применим формулу интегрирования по частям в интегралах, содержащих вторые производные функции $u$ и первые производные функций $u_{1}, u_{2}$. В итоге получим равенство

$$
\begin{aligned}
& -\int_{0}^{\pi} f \bar{u} d x_{3}=\mu\left(\int_{0}^{\pi}|\operatorname{grad} u|^{2} d x_{3}+\left(\left(\operatorname{Re} \xi_{1}\right)^{2}+\left(\operatorname{Re} \xi_{2}\right)^{2}-p_{1}^{2}-p_{2}^{2}\right) \int_{0}^{\pi}|u|^{2} d x_{3}\right) \\
& \quad+\mu\left(i\left(p_{1} \operatorname{Re} \xi_{1}+p_{2} \operatorname{Re} \xi_{2}\right) \int_{0}^{\pi}|u|^{2} d x_{3}\right)+(\lambda+\mu)\left(\int_{0}^{\pi}\left|\xi_{1} u_{1}+\xi_{2} u_{2}+i u_{3}^{\prime}\right|^{2} d x_{3}\right) .
\end{aligned}
$$

Для $p_{1}^{2}+p_{2}^{2}<1$ в вещественной части этого равенства применим оценки

$$
\begin{gathered}
\left|\int_{0}^{\pi} \operatorname{Re}(f \bar{u}) d x_{3}\right| \leqslant \frac{\varepsilon}{2} \int_{0}^{\pi}|u|^{2} d x_{3}+\frac{1}{2 \varepsilon} \int_{0}^{\pi}|f|^{2} d x_{3} \quad(\varepsilon>0), \\
\int_{0}^{\pi}|u|^{2} d x_{3} \leqslant \int_{0}^{\pi}|\operatorname{grad} u|^{2} d x_{3}
\end{gathered}
$$

и получим

$$
\int_{0}^{\pi}|\operatorname{grad} u|^{2} d x_{3}+\left(\left|\xi_{1}\right|^{2}+\left|\xi_{2}\right|^{2}\right) \int_{0}^{\pi}|u|^{2} d x_{3} \leqslant C_{1} \int_{0}^{\pi}|f|^{2} d x_{3} .
$$

Перенесем в системе (30) все слагаемые, не содержащие вторых производных функции $u$, в правую часть. Теперь нетрудно проверить, что для ограниченных по модулю $\xi_{1}, \xi_{2}$ и $p_{1}^{2}+p_{2}^{2}<1$ выполняется неравенство

$$
\|u\|_{2}^{2} \leqslant C\left(\|f\|_{0}^{2}+\|u\|_{1}^{2}\right) .
$$

Отсюда и из (31) следует априорная оценка

$$
\|u\|_{2}+\left(\left|\xi_{1}\right|^{2}+\left|\xi_{2}\right|^{2}\right)\|u\|_{0} \leqslant C_{2}\|f\|_{0}
$$

для ограниченных по модулю $\xi_{1}, \xi_{2}$ и $p_{1}^{2}+p_{2}^{2}<1$.

С учетом замечания 1 и неравенства (10) априорная оценка справедлива для всех $\xi_{1}$ и $\xi_{2}$, удовлетворяющих условию $p_{1}^{2}+p_{2}^{2}<1$. Таким образом, для $0 \leqslant \gamma<1$ к системе (28)-(29) применимы теоремы 1,4 .

\section{ЛитеРАТУРА}

[1] М. С. Агранович, М. И. Вишик, Эллиптические задачи с параметром и параболические задачи общего вида, УМН, 19:3 (1964), 53-161.

[2] S. Agmon, L. Nirenberg, Properties of solutions of ordinary differential equations in Banach space, Comm. Pure Appl. Math., 16:2 (1963), 121-239.

[3] Э. А. Коддингтон, Н. Левинсон, Теория обыкновенных дифференииалъных уравнений, Издательство ЛКИ, М., 2007.

[4] Б. А. Фукс, Теория аналитических функиий многих комплексных переменных, ОГИЗ, М., 1948.

[5] L. Nirenberg, H. F. Walker, The null spaces of elliptic partial differential operators in $\mathbb{R}^{n}$, J. Math. Anal. Apl., 42:2 (1973), 271-301.

[6] В. А. Кондратьев, Краевые задачи для параболических уравнений в замкнутых областях, в сб.: Труды ММО, т. 15, Изд-во МГУ, М., 1966, 400-452.

[7] Л. Хермандер, Анализ линейных дифференциалъных операторов с частными производными. Т. 3. Псевдодифберенциалъные операторы, Мир, М., 1987.

[8] С. А. Назаров, Б. А. Пламеневский, Эллиптические задачи в областях с кусочно гладкой гранищей, Наука, М., 1991. 
[9] М. С. Агранович, А. С. Дынин, Общие краевые задачи для эллиптических систем в многомерной области, ДАН, 146:3 (1962), 511-514.

[10] S. Agmon, A. Douglis, L. Nirenberg, Estimates near the boundary for solutions of elliptic partial differential equations satisfying general boundary conditions II, Comm. Pure Appl. Math., 17 (1964), 35-92.

[11] M. Schechter, Systems of partial differential equations in a half-space, Comm. Pure Appl. Math., 17 (1964), 423-434.

[12] C. B. Morrey, Multiple Integrals in the Calculus of Variations, Springer-Verlag, BerlinHeidelberg-New York, 1966.

[13] В. А. Кондратьев, Краевые задачи для эллиптических уравнений в областях с коническими или угловыми точками, в сб.: Труды ММО, т. 16, Изд-во МГУ, М., 1967, 219-292.

[14] Е. Янке, Ф. Эмде, Ф. Леш, Специалънъе функиии, Наука, М., 1977.

[15] И. Ц. Гохберг, М. Г. Крейн, Основные положения о дефектных числах, корневых числах и индексах линейных операторов, УМН, 12:2(74) (1957), 43-118.

Институт компьютерных технологий МЭСИ

e-mail: valnikishkin@yandex.ru

Поступило в редакцию

1 марта 2010 г. 